\title{
Wide-field optical coherence tomography angiography using extended field imaging technique to evaluate the nonperfusion area in retinal vein occlusion
}

\author{
This article was published in the following Dove Press journal: \\ Clinical Ophthalmology \\ 13 July 2016 \\ Number of times this article has been viewed
}

\section{Masayo Kimura \\ Miho Nozaki \\ Munenori Yoshida \\ Yuichiro Ogura}

Department of Ophthalmology and Visual Science, Nagoya City University Graduate School of Medical Sciences, Nagoya, Japan
Correspondence: Miho Nozaki Department of Ophthalmology and Visual Science, Nagoya City University Graduate School of Medical Sciences, I-Kawasumi, Mizuho-cho, Mizuho-ku, Nagoya, 467-860I, Japan

Tel +8I 52853825 I

Fax +8I 52 84I 9490

Email nozakim@med.nagoya-cu.ac.jp
Purpose: Optical coherence tomography angiography (OCTA) is a newly developed technology which allows us to reconstruct the three-dimensional chorioretinal vasculature without dye injection. OCTA is a noninvasive, rapid, and reproducible method to assess retinal ischemia. However, one of its limitations is the size of scanning area. A novel yet simple technique to expand the scan length on optical coherence tomography has been reported as an extended field imaging (EFI) technique. It involves imaging the posterior pole through trial frames fitted with $\mathrm{a}+20$ diopter lens. We applied this technique to OCTA to evaluate retinal vein occlusion.

Materials and methods: Ten eyes of nine patients with retinal vein occlusion were studied. The average age was 69.0 years (range: 49-93 years). We obtained OCTA images by using RTVue XR Avanti OCT with AngioVue ${ }^{\circledR}$. The images of OCTA with scan size of $8 \times 8 \mathrm{~mm}$ were obtained with and without EFI, and then they were compared.

Results: OCTA with EFI technique was performed successfully in all eyes. The nonperfusion area was well defined in superficial capillary plexus layer. The images with EFI were able to capture the larger area of the fundus by an average of $188.5 \%$ than those without EFI. The posterior pole inside the vascular arcade was well covered with this technique. The area of the fundus imaged by OCTA with EFI technique was even larger than that of fluorescein angiography using Heidelberg Retina Angiograph 2, which captured a $30^{\circ}$ field.

Conclusion: Our results suggested that OCTA with EFI technique is very useful to evaluate the retinal ischemia in retinal vein occlusion.

Keywords: optical coherence tomography angiography, OCTA, retinal vein occlusion, RVO, nonperfusion area, wide-field imaging, extended field imaging, EFI

\section{Introduction}

Optical coherence tomography angiography (OCTA) is a newly developed technology which allows us to reconstruct the three-dimensional chorioretinal vasculature without dye injection. ${ }^{1-4}$ It is a noninvasive, rapid, and reproducible method to assess retinal ischemia. ${ }^{3,4}$ However, one of its limitations is the size of scanning area. AngioVue ${ }^{\circledR}$ system (RTVue XR Avanti OCT; Optovue, Inc., Fremont, CA, USA) is commercially available now, but its maximum scanning size of $8 \times 8 \mathrm{~mm}$ is not enough to replace fluorescein angiography (FAG).

A novel yet simple technique to expand the scan length on optical coherence tomography (OCT) has been reported as an extended field imaging (EFI) technique, ${ }^{5}$ 
which involves imaging the posterior pole through trial frames fitted with a +20 diopter lens.

In this study, we sought to further explore the feasibility of EFI technique to OCTA for evaluating the nonperfusion area due to retinal vein occlusion (RVO).

\section{Methods}

\section{Patients}

This study was a prospective study of ten eyes from nine patients with RVO who visited the Nagoya City University Hospital between May 2015 and November 2015. This study was approved by the Institutional Review Board of the Nagoya City University Graduate School of Medical Sciences and was conducted in accordance with the ethical standards stated in the 1964 Declaration of Helsinki. The eyes with poor quality of the OCT images due to cataract or poor fixation and the eyes with epiretinal membrane were excluded from the study. Written consent was obtained from all patients.

\section{OCTA with or without EFI technique}

The images of OCTA with scanning size of $8 \times 8 \mathrm{~mm}$ were obtained with and without EFI technique by using RTVue XR Avanti OCT with AngioVue ${ }^{\circledR}$, and then the expansion rates were calculated using ImageJ software (NIH) (http:// imagej.nih.gov/ij/).

EFI technique was performed as described previously (Figure 1). ${ }^{5}$

If the eyes showed retinal nonperfusion (RNP) in the posterior pole by FAG, we also measured the area of RNP using ImageJ sortware.

\section{Results}

\section{Patients' characteristics}

A total of ten eyes from nine patients (age, 49-93 years [69.0 12.5 years]; four males and five females) with RVO

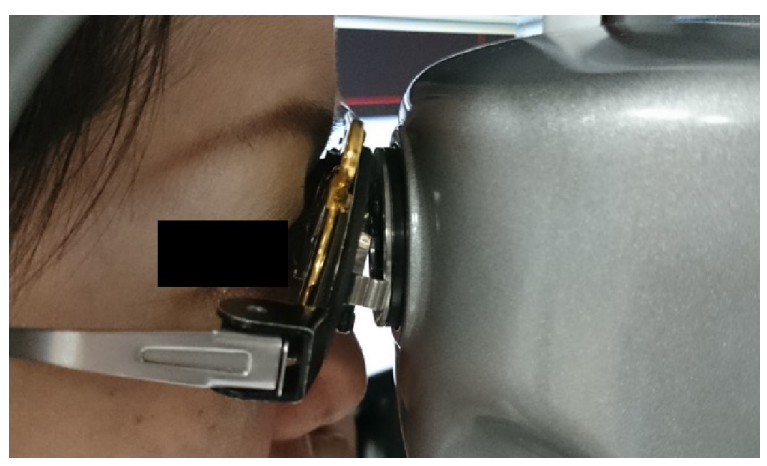

Figure I Extended field imaging (EFI) technique.

Note: The +20 diopter trial lens is set into trial frame, and a subject is undergoing EFI optical coherence tomography angiography (OCTA) imaging with the RTVue XR Avanti OCT. were evaluated in this study. Patient classification included eight eyes with branch retinal vein occlusion and two eyes with central retinal vein occlusion. Seven eyes did not have macular edema, but two eyes had macular edema. The duration of disease history was 20 \pm 19.6 months (4-72 months), and average LogMAR visual acuity was $0.01 \pm 0.16$ ( -0.18 to 0.40 ). Nine of ten eyes were also evaluated by FAG, and four of nine eyes were examined using both Heidelberg Retina Angiograph 2 (HRA2; Heidelberg Engineering, Heidelberg, Germany) and Optos 200Tx (Optos PLC, Dunfermline, UK), and five eyes were evaluated only by Optos 200Tx. One patient who was not examined by FAG was 93 years old, and due to a previous history of hypertension and angina, dye-injection angiography was avoided.

\section{EFI technique}

In all ten eyes of nine patients, clear images were obtained with EFI technique. The images with EFI were able to capture a larger area of the fundus by an average of $188.5 \% \pm 3.6 \%$ (range: 180.1\%-191.9\%) than those without EFI (Figure 2), and it was even larger than the image of FAG using HRA2, which captured a $30^{\circ}$ field (Figure 3 ).

The area of RNP due to RVO was well defined in the superficial capillary plexus layer in all cases even with EFI technique. In four of nine eyes which had RNP in posterior pole, the average area of RNP was $18.3 \pm 8.8 \mathrm{~mm}^{2}$ with EFI technique and $16.8 \pm 8.5 \mathrm{~mm}^{2}$ with FAG. However, due to low resolution of images, the area of RNP was not able to be measured in deep capillary plexus layer (Figure 4).

\section{Discussion}

The EFI technique is a novel yet simple technique to expand the scan length on OCT developed by Uji and Yoshimura. ${ }^{5}$ It involves the imaging of the posterior pole through trial frames fitted with a +20 diopter lens. In this study, we applied this technique to OCTA to evaluate RVO and found that the EFI technique allowed us to evaluate a larger field than what a commercially available OCTA system can offer.

OCTA is a noninvasive, rapid, and reproducible method to assess chorioretinal microcirculation without dye injection. However, one of its limitations is the size of scanning area for now. RTVue XR Avanti OCT is the first commercially available OCTA machine, but the maximum scan size of $8 \times 8$ $\mathrm{mm}$ is not adequate to evaluate retinal diseases, such as diabetic retinopathy and RVO. Since RNP is highly associated with pathogenesis of macular edema in diabetic retinopathy and RVO, detecting RNP would be crucial to predict the prognosis of the diseases and making therapeutic strategies for macular edema. ${ }^{6,7}$ 


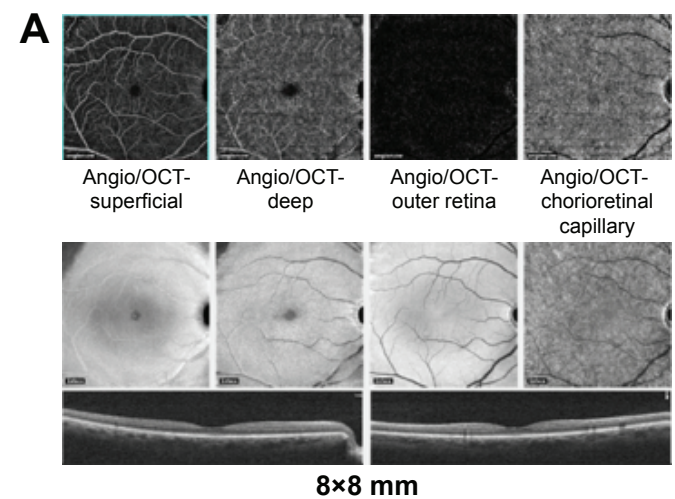

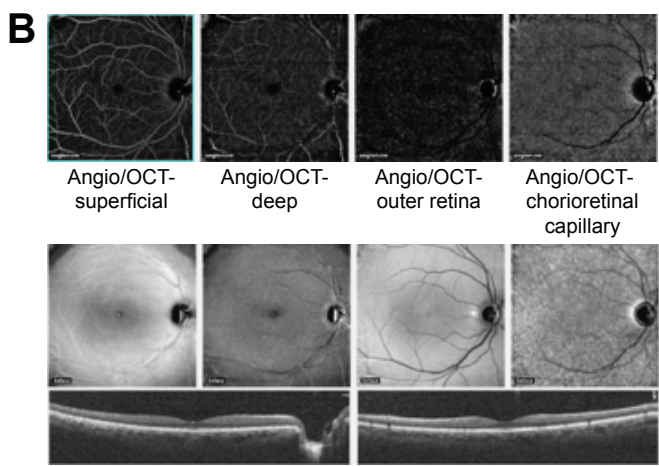

$8 \times 8 \mathrm{~mm}$ with EFI technique

Figure 2 OCTA $(8 \times 8 \mathrm{~mm})$ images with or without EFI technique (normal subject).

Notes: (A) Regular OCTA image $(8 \times 8 \mathrm{~mm})$ does not cover the arcade vessels or optic disc. (B) OCTA image $(8 \times 8 \mathrm{~mm})$ with EFI technique showed the arcade vessels and the entire optic disc.

Abbreviations: $\mathrm{EFI}$, extended field imaging; OCTA, optical coherence tomography angiography.

In our study, OCTA with EFI technique can capture the image larger than the image by FAG using HRA2, which captured a $30^{\circ}$ field.

Other OCTA machines, which are now available, can create "montage" images of OCTA based on the several regular scan sizes of OCTA images, to evaluate a wider field. However, this requires multiple capture of various areas, and it is necessary to involve patients' cooperation and concentration. In contrast, the EFI technique, which involves imaging the posterior pole through trial frames fitted with $a+20$ diopter lens, is very simple and a single capture would be enough to evaluate RNP. In our current study, in all ten eyes of nine patients, clear images were obtained with EFI technique. And, for a 93-year-old male who had hypertension and angina, FAG was avoided, but he was able to tolerate
OCTA with EFI technique. From our findings, OCTA with EFI technique may be beneficial in daily clinic practice to follow up RVO.

The limitations of our present study include the fact that this was a study that only included a limited number of patients. Furthermore, EFI results in a decrease in image resolution because EFI does not change the hardware capabilities of the OCT system. It simply magnifies each pixel, so the detailed information, such as microaneurysms or venous shunt, is missed in the $8 \times 8 \mathrm{~mm}$ OCTA images with EFI technique. However, the resolution of the OCTA images with EFI technique was adequate for evaluating the area of RNP.

In conclusion, our results suggested that OCTA with EFI technique is very useful and practical for evaluating retinal ischemia in RVO.

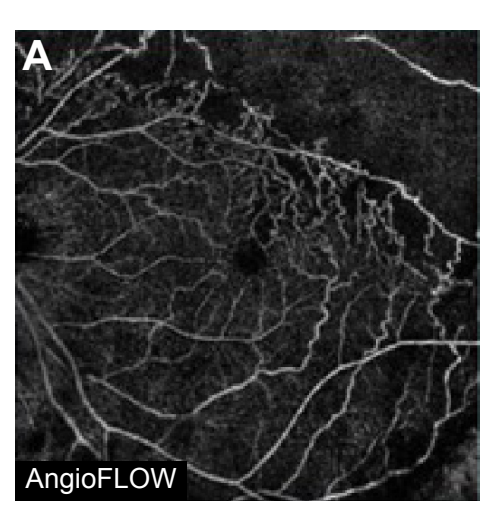

$8 \times 8 \mathrm{~mm}$

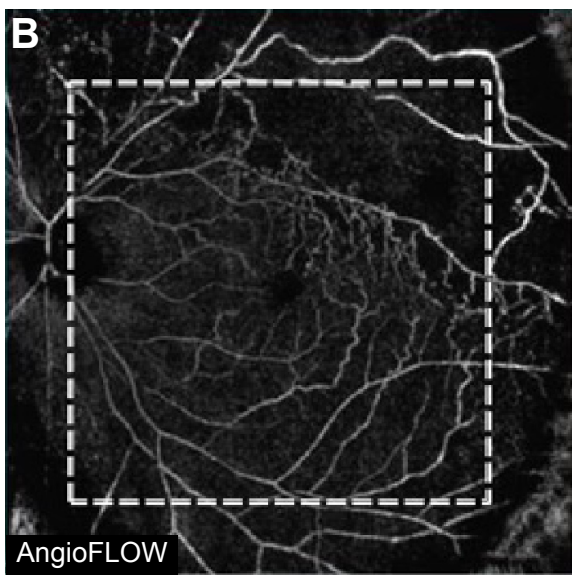

$8 \times 8 \mathrm{~mm}$ with EFI technique

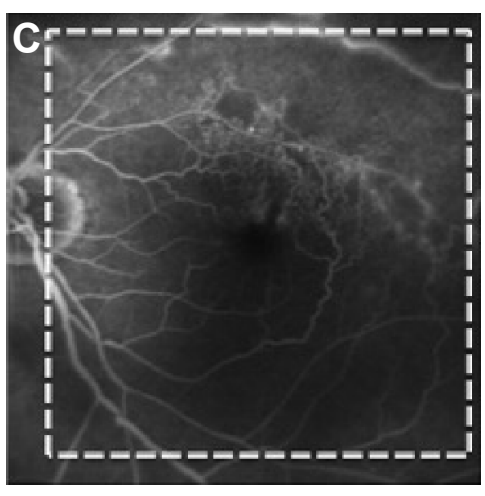

FAG $\left(30^{\circ}\right)$ using HRA2

Figure 3 Representative case of a 56-year-old male with BRVO.

Notes: (A) Regular OCTA image in superficial retinal capillary layer $(8 \times 8 \mathrm{~mm})$. (B) OCTA image $(8 \times 8 \mathrm{~mm})$ in superficial capillary plexus layer with EFI technique. (C) FAG image obtained by HRA2 $\left(30^{\circ}\right)$. White dot square represents the same area with regular OCTA image $(8 \times 8 \mathrm{~mm})(\mathbf{A})$. OCTA image with EFI technique can capture an even larger area than the image of FAG using HRA2 (B).

Abbreviations: BRVO, branch retinal vein occlusion; EFI, extended field imaging; FAG, fluorescein angiography; HRA2, Heidelberg Retina Angiograph 2; OCTA, optical coherence tomography angiography. 


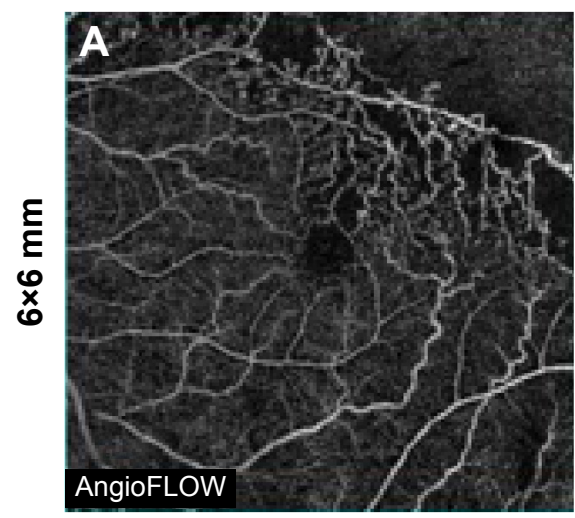

SSADA - superficial

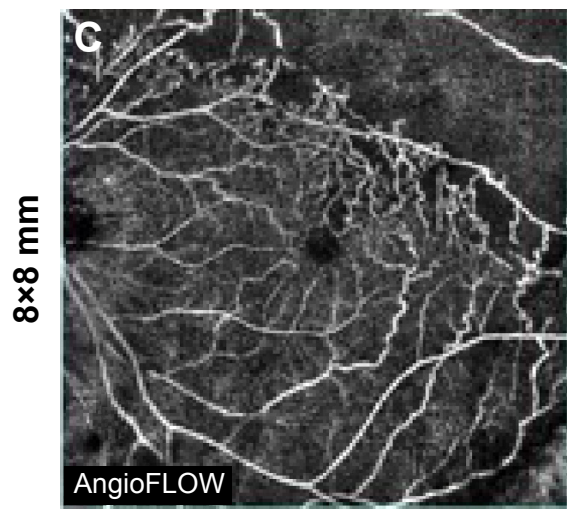

SSADA - superficial

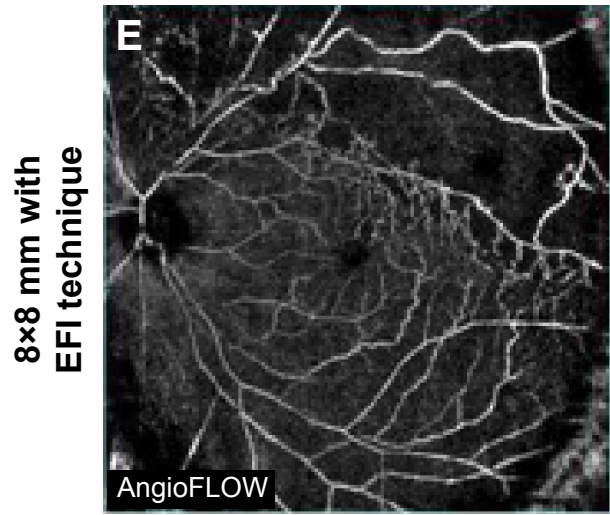

SSADA - superficial

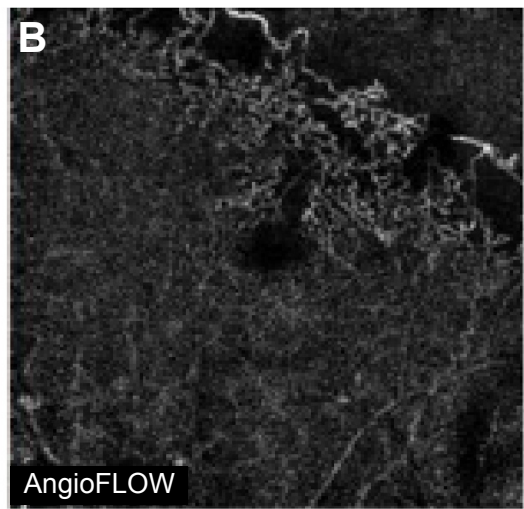

SSADA - deep

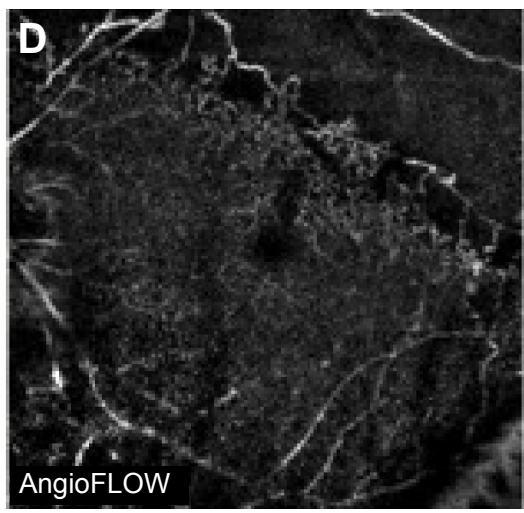

SSADA - deep

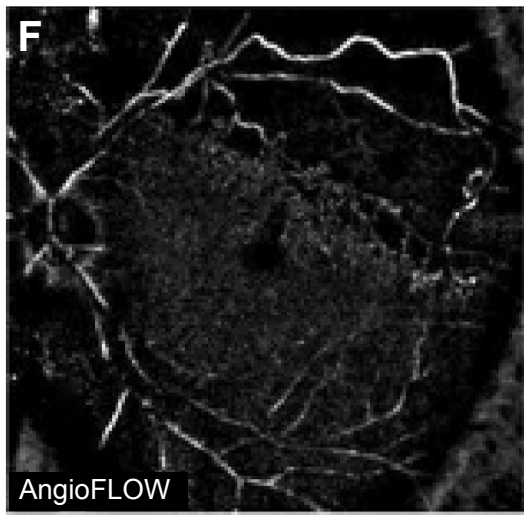

SSADA - deep

Figure 4 Comparison of image resolution between scan size and retinal capillary plexus layer.

Notes: (A) Regular OCTA image in superficial capillary plexus layer $(6 \times 6 \mathrm{~mm})$. (B) Regular OCTA image in deep capillary plexus layer (6×6 mm). (C) Regular OCTA image in superficial capillary plexus layer $(8 \times 8 \mathrm{~mm})$. (D) Regular OCTA image in deep capillary plexus layer $(8 \times 8 \mathrm{~mm})$. (E) OCTA image in superficial capillary plexus layer $(8 \times 8 \mathrm{~mm})$ with EFI technique. (F) OCTA image in deep capillary plexus layer $(8 \times 8 \mathrm{~mm})$ with EFI technique. Regular OCTA images showed retinal nonperfusion clearly, both in superficial capillary plexus layer $(\mathbf{A}, \mathbf{C})$ and deep capillary plexus layer $(\mathbf{B}, \mathbf{D})$ and vessel abnormalities, such as vessel dilation and shunt formation, are also visible in both plexus layers (A-D). However, the retinal nonperfusion is hardly visible in OCTA image in deep capillary plexus layer $(8 \times 8 \mathrm{~mm})$ with $\mathrm{EFI}$ technique $(\mathbf{F})$. Only superficial capillary plexus layer in OCTA image $(8 \times 8 \mathrm{~mm})$ with EFI technique can be used to evaluate retinal nonperfusion, but not detailed vessel abnormalities (E).

Abbreviations: EFI, extended field imaging; OCTA, optical coherence tomography angiography; SSADA, split-spectrum amplitude decorrelation angiography.

\section{Acknowledgment}

The authors thank Fumie Shibuya for assisting with extended field imaging technique.

\section{Disclosure}

The authors report no conflicts of interest in this work.

\section{References}

1. Jia Y, Tan O, Tokayer J, et al. Split-spectrum amplitude-decorrelation angiography with optical coherence tomography. Opt Express. 2012; 20(4):4710-4725.

2. Takase N, Nozaki M, Kato A, Ozeki H, Yoshida M, Ogura Y. Enlargement of foveal avascular zone in diabetic eyes evaluated by en face optical coherence tomography angiography. Retina. 2015;35(11): 2377-2383. 
3. Suzuki N, Hirano Y, Yoshida M, et al. Microvascular abnormalities on optical coherence tomography angiography in macular edema associated with branch retinal vein occlusion. Am J Ophthalmol. 2016;161: 126-132.

4. Coscas F, Glacet-Bernard A, Miere A, et al. Optical coherence tomography angiography in retinal vein occlusion: Evaluation of superficial and deep capillary plexa. Am J Ophthalmol. 2016;161:160-171.

5. Uji A, Yoshimura N. Application of extended field imaging to optical coherence tomography. Ophthalmology. 2015;122(6):1272-1274.
6. Takamura Y, Tomomatsu T, Matsumura T, et al. The effect of photocoagulation in ischemic areas to prevent recurrence of diabetic macular edema after intravitreal bevacizumab injection. Invest Ophthalmol Vis Sci. 2014;55(8):4741-4746.

7. Noma H, Funatsu H, Yamasaki M, et al. Pathogenesis of macular edema with branch retinal vein occlusion and intraocular levels of vascular endothelial growth factor and interleukin-6. Am J Ophthalmol. 2005; 140(2):256-261.
Clinical Ophthalmology

\section{Publish your work in this journal}

Clinical Ophthalmology is an international, peer-reviewed journal covering all subspecialties within ophthalmology. Key topics include: Optometry; Visual science; Pharmacology and drug therapy in eye diseases; Basic Sciences; Primary and Secondary eye care; Patien Safety and Quality of Care Improvements. This journal is indexed on

Submit your manuscript here: http://www.dovepress.com/clinical-ophthalmology-journal

\section{Dovepress}

PubMed Central and CAS, and is the official journal of The Society of Clinical Ophthalmology (SCO). The manuscript management system is completely online and includes a very quick and fair peer-review system, which is all easy to use. Visit http://www.dovepress.com/ testimonials.php to read real quotes from published authors. 